\title{
Mechanical properties of plasterboards: experimental tests and statistical analysis
}

\author{
Crescenzo Petrone ${ }^{1}$, Gennaro Magliulo², Gaetano Manfredi ${ }^{3}$ \\ Department of Structures for Engineering and Architecture, University of Naples Federico II, Italy
}

\begin{abstract}
Plasterboard components are widely used in current buildings worldwide. Despite their extensive use, the lack of a comprehensive test campaign on plasterboards in the current literature is denoted. An extensive test campaign, consisting of 302 tests, on plasterboards is performed both in tension and compression. A set of five plasterboard typologies is selected. The tests are performed in two different load directions, i.e. parallel or transversal to the direction of production. Tensile strength of boards is systematically smaller than compressive strength, whereas elastic modulus values in compression and in tension are similar. Two different regression laws are defined, matching compression and tension behavior, respectively. An orthotropic behavior is exhibited in case the boards are loaded in tension. The significant influence of board thickness on their mechanical properties is also highlighted. Finally the most appropriate probability distribution function is estimated for several mechanical parameters and the corresponding data dispersion is evaluated. The performed activities can be used as reference for future numerical studies involving plasterboards.
\end{abstract}

Keywords: plasterboard, gypsum, tension test, compression test, uncertainty, regression

\footnotetext{
${ }^{1}$ Research Associate. Department of Civil, Environmental \& Geomatic Engineering. University College London. London WC1E 6BT. Email: c.petrone@ucl.ac.uk

${ }^{2}$ Assistant Professor. Department of Structures for Engineering and Architecture, University of Naples Federico II, via Claudio, 21, 80125, Naples, Italy. E-mail: gmagliul@unina.it.

${ }^{3}$ Full Professor. Department of Structures for Engineering and Architecture, University of Naples Federico II, via Claudio, 21, 80125, Naples, Italy. E-mail: gamanfre@ unina.it.
} 


\section{INTRODUCTION}

Plasterboard components are widely used in current buildings worldwide. Plasterboards are employed for partitions, wall lining and ceilings. Nowadays, the use of plasterboards is increasing both in Eastern and Western Europe; in these regions there are currently more than 200 factories producing plasterboards (Eurogypsum 2014). Plasterboards are composed of a plaster core encased in, and firmly bonded to, paper liners to form flat rectangular boards (CEN 2004a). The properties of these materials, particularly the gypsum, can be modified to meet specific requirements, such as fire resistance, humidity resistance, impact resistance, etc. (e.g. (Gencel et al. 2014)).

Plasterboards are used for both structural (Memari and Solnosky 2014; Peterman and Schafer 2014; Serrette and Ogunfunmi 1996; Serrette et al. 1997) and nonstructural (Lee et al. 2007; Magliulo et al. 2012; Magliulo et al. 2014; Petrone et al. 2015b; Retamales et al. 2013) walls: they are screwed either to the flanges of steel studs or to wood studs. Plasterboard components may significantly influence the performance of the walls. A dynamic test on a six-story timber framed building during its construction (Ellis and Bougard 2001) demonstrated that the addition of internal plasterboards resulted in increased natural frequencies of the building, due to their contribution to the lateral stiffness. An experimental study (Telue and Mahendran 2004) showed that the strength of the studs in compression significantly increased when they were lined with plasterboards. A finite element model was also defined and validated using experimental results. A numerical study on the contribution of plasterboards to the structural performance of multi-story light wood frame buildings (Asiz et al. 2011) also evidenced that they lead to stiffer structures and smaller drifts (up to 30\%). Petrone et al. (2015a) demonstrated the significant contribution of the plasterboards in the out-of-plane seismic behavior of plasterboard partitions through quasi-static tests.

The mechanical properties, e.g. modulus of elasticity or tensile/compressive strength, of the plasterboards may assume a key role in the whole performance of a building. Numerical models (Telue and Mahendran 2004; Asiz et al. 2011) of building components which include plasterboard elements require the definition of the mechanical properties of plasterboards. Finally their mechanical properties also influence the acoustic performance and modelling of plasterboard components (Arjunan et al. 2013), such as internal partitions. Very limited studies are available in literature concerning the mechanical properties of plasterboard partitions, despite their increasing importance in different areas of civil engineering. Compressive tests aiming at 
assessing the mechanical properties of an innovative gypsum board for thermal insulation purposes were included in (Oliver-Ramirez et al. 2011). Some out-of-plane four-point bending tests on plasterboards are performed in Bouterf et al. (2015). However, the lack of a comprehensive test campaign on plasterboards in the current literature is denoted. Existing numerical studies employ the mechanical properties resulting from a minimal number of tests; furthermore, the uncertainty related to this material is not taken into account, due to the limited number of the available tests.

An extensive test campaign, consisting of 302 tests, is performed aiming at evaluating compression and tension behavior of plasterboards. A set of five plasterboard typologies is selected. The resulting tensile and compressive strengths, as well as the elastic moduli in tension and compression are assessed for each plasterboard typology. Two different regression laws are defined matching compression and tension behavior, respectively, of plasterboards. The influence of some parameters, such as the thickness of boards and the direction of loading, on the mechanical properties is assessed. Finally the most appropriate distribution function for several mechanical parameters is estimated and the corresponding data dispersion is evaluated.

The estimated parameters can be used as reference material for future numerical studies involving plasterboards.

\section{EXPERIMENTAL STUDY}

The tests, both in tension and compression, were carried out with the aim of determining the mechanical properties of gypsum plasterboards. Both tensile and compression tests were performed according to EN 789 (CEN 2004b). Tension and compression tests were carried out on different boards:

- $\quad 12.5 \mathrm{~mm}$ thick and $18 \mathrm{~mm}$ thick standard plasterboards, named 12SB and 18SB in the following. These gypsum boards are used for general installations with metal studs for partitions, lining systems and ceilings. They are characterized by gypsum plaster with density equal to $720 \mathrm{~kg} / \mathrm{m}^{3}$ and $862 \mathrm{~kg} / \mathrm{m}^{3}$, respectively.

- $\quad 12.5 \mathrm{~mm}$ thick, $15 \mathrm{~mm}$ thick and $18 \mathrm{~mm}$ thick high density plasterboards, named 12HDB, 15HDB and 18HDB in the following, respectively. These high density core gypsum boards are stronger, harder and heavier than standard plasterboards, providing better fire, impact and acoustic resistance. In particular, their density is $831 \mathrm{~kg} / \mathrm{m} 3893 \mathrm{~kg} / \mathrm{m} 3$ for $12 \mathrm{HDB}$ and $15 \mathrm{HDB}$, respectively, whereas $18 \mathrm{HDB}$ is characterized by a $920 \mathrm{~kg} / \mathrm{m} 3$ density. Then these solutions are typically adopted for 
installations with stud partitions, lining systems and ceilings that require superior fire, impact and acoustic resistance.

Paper liners are characterized by the same properties for the different boards. Their specific mass is in the range $180-200 \mathrm{~g} / \mathrm{m}^{2}$. The plasterboards are representative of the most common plasterboards adopted in Europe for both ceiling and partition systems.

A total number of 302 tests were performed (Table 1) for the above mentioned plasterboards and for two different load directions, i.e. parallel or perpendicular to the direction of production. It is expected that the mechanical properties are different in the two orthogonal directions. The amount of tests for each board is not constant since some tests were excluded from the database due to errors in the data acquisition.

In the following paragraphs, the code requirements for plasterboards are firstly highlighted; then, the experimental test setups for tensile and compressive tests are described.

\begin{tabular}{c|cccc}
\hline \multicolumn{2}{c}{ Longitudinal } & & \multicolumn{2}{c}{ Transversal } \\
\hline & Compressive & Tensile & Compressive & Tensile \\
\hline 12SB & 14 & 15 & 16 & 16 \\
\hline 18SB & 16 & 16 & 15 & 15 \\
\hline 12HDB & 14 & 15 & 15 & 15 \\
\hline 18HDB & 15 & 15 & 16 & 16 \\
\hline
\end{tabular}

Table 1. Number of tests for each board typology.

\section{Code requirements for plasterboards}

EN 520 (CEN 2004a) defines the requirements that gypsum plasterboards should meet: thermal insulation properties, fire resistance, acoustic properties, permeability to both air and water and mechanical properties. From a mechanical point of view, this code provides that flexural strength of the board and shear strength of screw connections in the boards should be checked. However, these tests would not allow a reliable and direct estimation of both compressive and tensile properties of the board itself.

Tension and compression tests were therefore performed according to EN 789 (CEN 2004b), which rules the assessment of mechanical properties of wood-based panels. These tests were carried out on plasterboards that meet the prescriptions included in EN 520. The adopted test methods for both tension and compression tests are described in the two following sections. 


\section{Tension tests}

The EN 789 standard tensile test consists in applying a tensile stress in the longitudinal direction of the specimen until failure occurs. The specimens are shaped in order to subject the central portion to pure tension stresses. They are obtained by properly shaping a single plasterboard (Figure 1), obtaining a $3 \mathrm{~cm}$ wide central portion. The objective is to determine the board elastic modulus, strength and ultimate strain.

The tests were performed in displacement-control: a monotonically increasing displacement is applied with a $0.5 \mathrm{~mm} / \mathrm{min}$ velocity. Two displacement transducers (LVDT sensors) were placed on two opposite faces of the specimen; they measure the deformation of a $200 \mathrm{~mm}$ long portion of the specimen (Figure 1). Metallic fixing supports were glued on each side of the board, in order to position the instrumentation on the specimen (Figure 1). The applied load was recorded by means of a load cell.

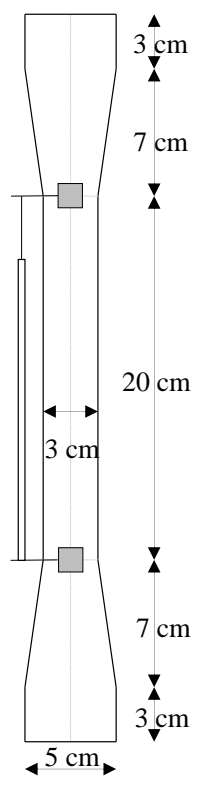

(a)

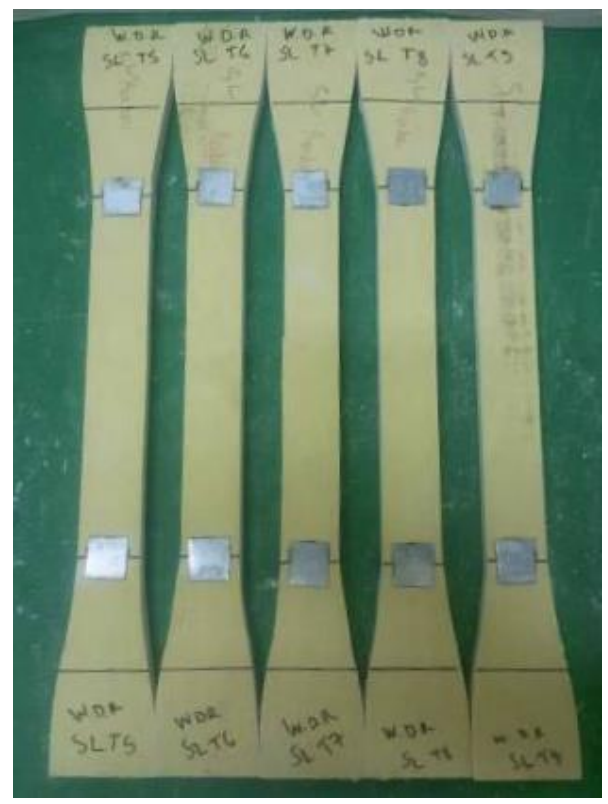

(b)

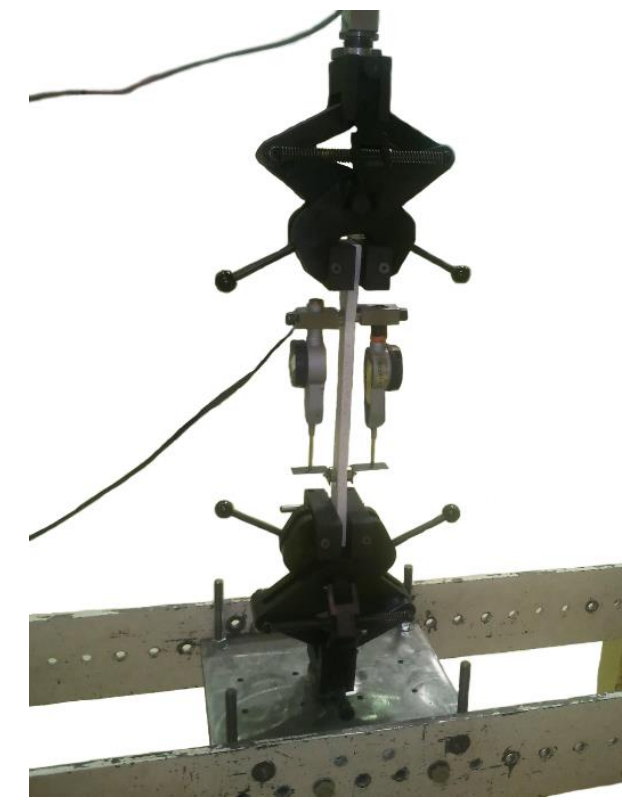

(c)

Figure 1. Specimen for tension tests: (a) geometry, (b) photo and (c) test setup.

\section{Compression tests}

Compression tests were also performed according to the EN 789 standard. The specimens consist of four boards perfectly glued together (Figure 2), in order to avoid that buckling of the boards dominates the failure; these four boards are obtained from a single plasterboard. A glue suitable for bonding gypsum dry flooring elements is adopted. This test consists in applying a compression stress in the longitudinal direction of the specimen until failure occurs. The objective is the assessment of compression elastic modulus, strength and ultimate strain. 
The tests were performed in displacement-control: a monotonically increasing displacement was applied with a $1.0 \mathrm{~mm} / \mathrm{min}$ velocity. Two displacement transducers (LVDT sensors) were placed on two opposite sides of the specimen, measuring the deformation of a $100 \mathrm{~mm}$ portion of the specimen. The applied load was recorded by means of a load cell.

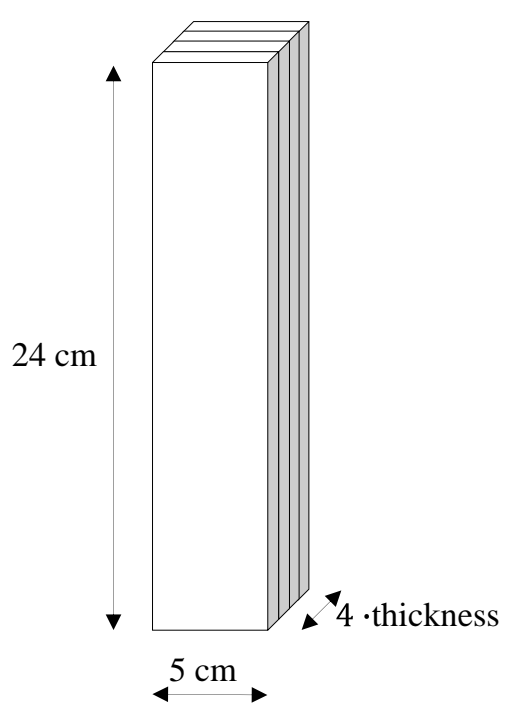

(a)

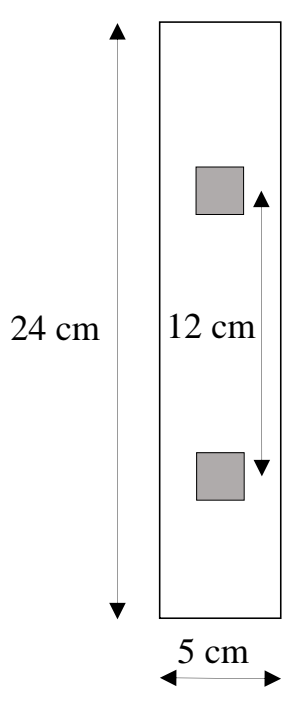

(b)

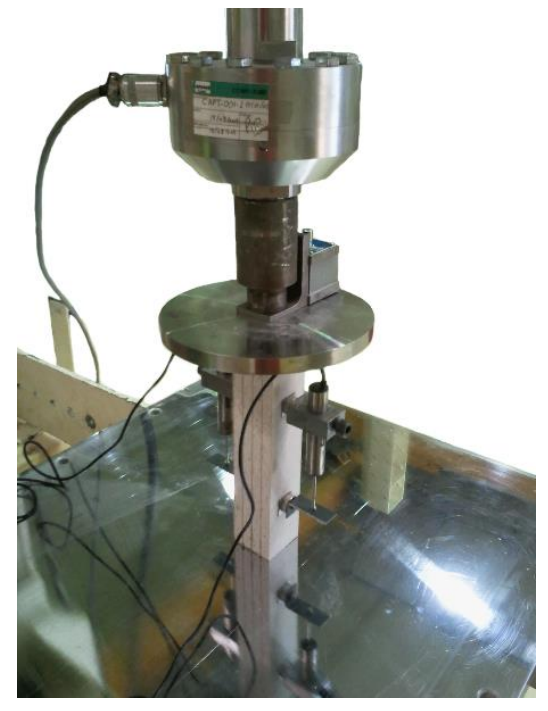

(c)

Figure 2. Specimen for compression tests: (a) global assembly, (b) geometry and (c) test setup.

\section{RESULTS AND DISCUSSION}

\section{Damage description}

Both compression and tension tests (Table 1) were performed until the failure of the specimen was recorded. Tension tests typically caused the specimens to exhibit a sub horizontal crack both in gypsum and in paper (Figure 3).

In compression tests, boards typically exhibited a sub-vertical crack in their central portion along with inclined cracks close to their boundaries (Figure 4a). The inclined cracks were caused by the friction forces at the interface between the loading surface and the specimen; this pattern is very similar to typical crack patterns in concrete cubes in compression. Moreover, in very few cases adjacent boards detached, due to the failure of the glue layer (Figure 4b). These tests are removed from the database, since the collapse of the specimen is not recorded and the recorded deformation is associated to the glue layer failure. The removal of these tests is performed by means of an outlier analysis, which is detailed in the following sections. 


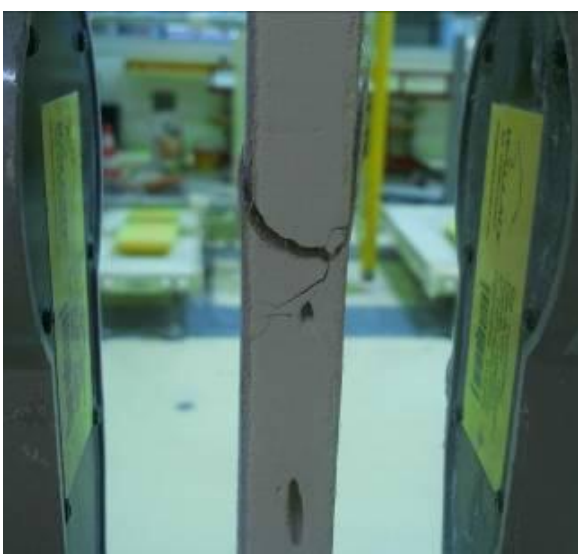

(a)

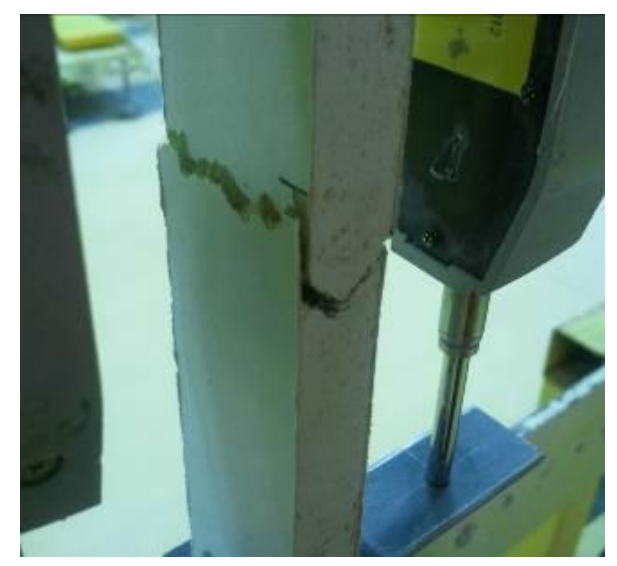

(c)

Figure 3. Tension tests: (a-b) typical failure modes.

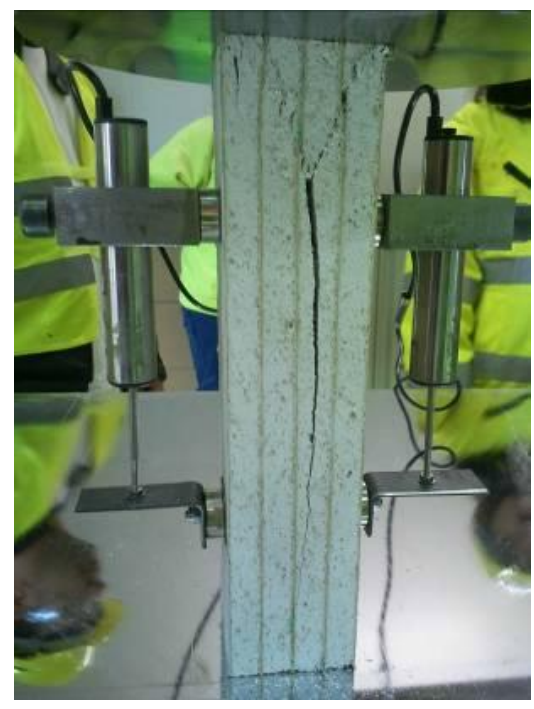

(a)

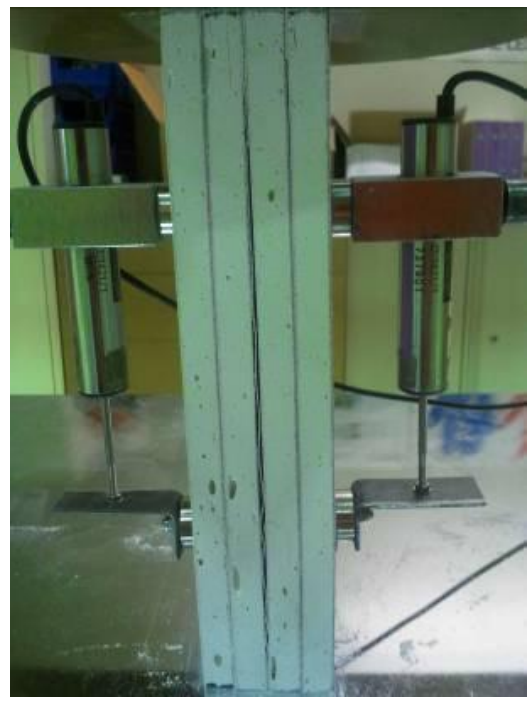

(b)

Figure 4. Compression tests: (a) typical failure mode and (b) failure of glue among adjacent boards.

\section{Data processing}

As mentioned above, each board was tested both in tension and in compression in its longitudinal and transversal direction. For each test a force displacement diagram can be obtained. The force was recorded by a load cell, whereas the displacement is evaluated as the mean displacement recorded by the two displacement transducers positioned at the two opposite sides of the specimen. Stress-strain diagrams are then plotted, considering both the actual area of the tested specimen and the actual measuring length of the displacement transducers. The diagrams are plotted up to the failure of the specimens. The ultimate strain is evaluated as the strain corresponding to a $20 \%$ stress drop with respect to the maximum recorded stress.

The stress-strain relationships resulting from the different specimens in tension are plotted in Figure 5 for each plasterboard typology, whereas the stress-strain relationships in compression are included in Figure 6. The 
board typology is included in the graph, where "L" e "T" suffixes denote whether the test was performed in the longitudinal direction, i.e. direction of production of the boards, or in the transversal direction, respectively. Some comments can be drawn from the comparison of the stress-strain relationships in compression and in tension.

- The specimens show a ductile behavior in tension with a more brittle behavior in compression; the ultimate tensile strain is much larger than the ultimate compressive strain. Moreover, the tensile strength is systematically smaller than the compressive strength. The shape of the curves also differs significantly: tension stress-strain diagrams show a marked yielding point, suggesting that these diagrams can be properly schematized as bilinear; compression curves, instead, do not exhibit any clear yielding point. These features are probably related to the presence of the paper, whose contribution is significant when the specimen is loaded in tension and is negligible in case it is loaded in compression.

- A more brittle behavior is exhibited in the transversal direction compared to the longitudinal direction, in case the specimens are loaded in tension; a smaller strength is also recorded in the transversal direction, clearly underlining the orthotropic behavior exhibited by the tested plasterboards. Compression behavior is not much influenced by the testing direction, since the paper contribution is negligible in case the specimen is loaded in compression.

- The comparison among boards with different thicknesses, i.e. $12 \mathrm{SB}$ vs $18 \mathrm{SB}$ and $12 \mathrm{HDB}$ vs $15 \mathrm{HDB}$ vs $18 \mathrm{HDB}$, highlights that the larger the thickness, the larger the compressive strength; this outcome may be justified by (a) the density and/or (b) crack propagation for different board thicknesses. In particular, it should be noted that density increases with board thickness, for the considered specimens. The comparison between 12SB (18SB) and 12HDB (18HDB) highlights a larger compressive strength in high density boards.

- Finally, some outliers in the recorded data are clearly visible in many stress-strain plots. They may have been caused by some errors in the preparation of the specimen, in the application of loads, etc.. The need to perform an outlier analysis, in order to remove data characterized by mistakes, is therefore claimed. 

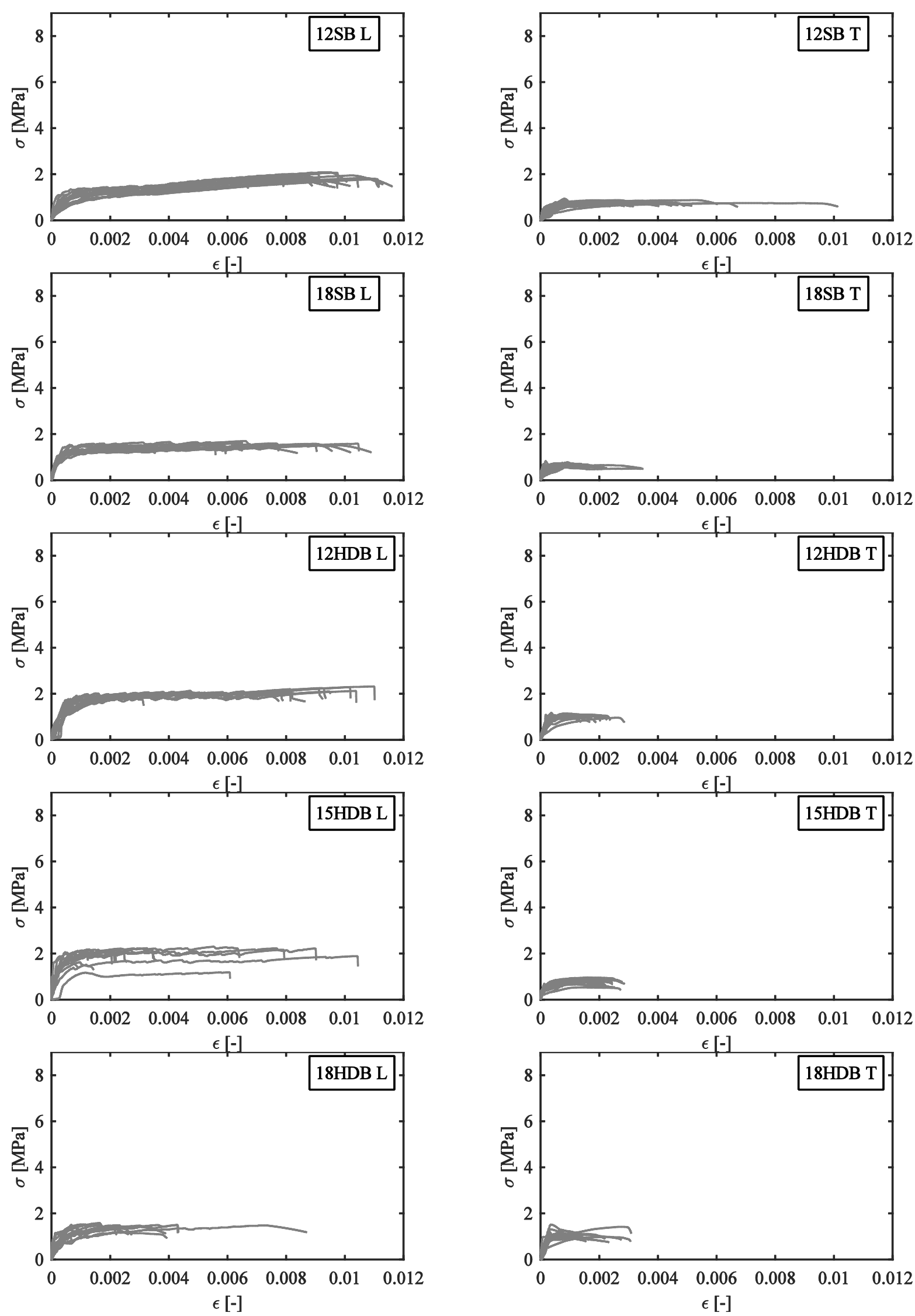

Figure 5. Stress-strain diagrams resulting from tension tests on the plasterboards. 

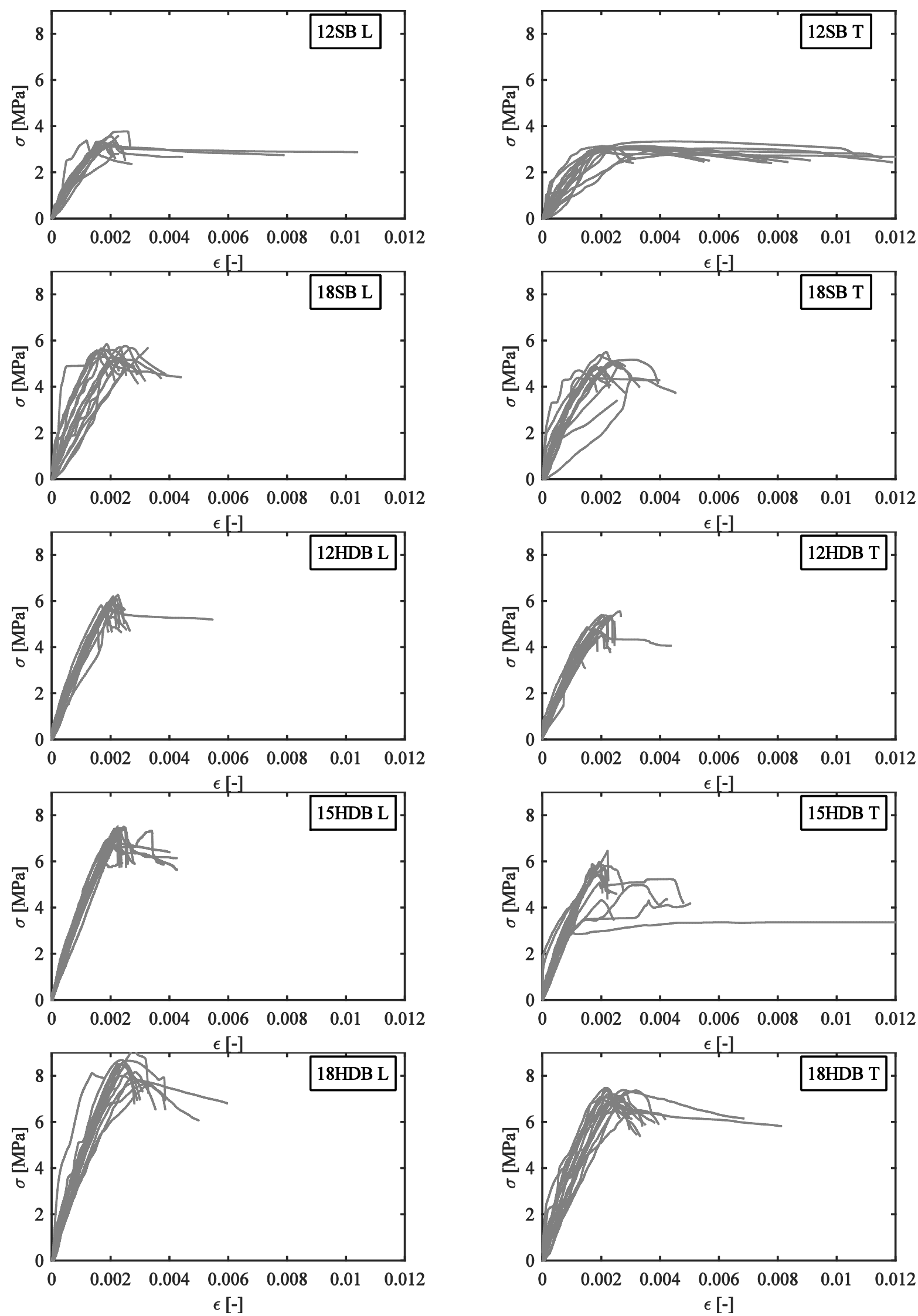

Figure 6. Stress-strain diagrams resulting from compression tests on the plasterboards. 


\section{Regression analysis}

This section deals with the identification of regression laws that can be employed to model both compression and tension behavior of plasterboards. This task would be useful for future implementations of the actual stressstrain relationship in different applications, e.g. FEM analysis of shear stud wall panels.

Before performing the regression analysis, the outliers should be removed from the database. Indeed, in Figure 5 and Figure 6 some outliers can be clearly found. These outliers may have been caused by several mistakes, e.g. improper mounting of the specimen and consequent incorrect application of loads, errors in the measurements, uneven contact surface, etc.

The outlier analysis is performed on two different parameters: the maximum strength $f_{c}\left(f_{t}\right)$ and the initial stiffness $E_{c}\left(E_{t}\right)$, for compression (tension) tests. The modulus of elasticity $E_{c}$ is evaluated as the secant stiffness from $10 \% f_{c}$ to $40 \% f_{c}$ :

$$
E_{c}=\left(0.4 f_{c}-0.1 f_{c}\right) /\left(\epsilon_{0.4 f c}-\epsilon_{0.1 f c}\right)
$$

according to the provisions included in EN 789. The modulus of elasticity in tension $E_{t}$ is evaluated in a similar fashion.

The modified Thompson's Tau technique (Cimbala 2011) is employed, assuming a student $t$-value evaluated at $\alpha=0.01$. Figure 7 shows an outlier identification for 15HDB boards, when subjected to tensile loads in their longitudinal direction. The outliers are denoted with a red circle in Figure $7 \mathrm{a}-\mathrm{b}$, whereas the mean is highlighted with a black line and the maximum allowed residuals are highlighted with red lines. Finally, the stress-strain relationships which were removed from the database are depicted in red in Figure 7c. 


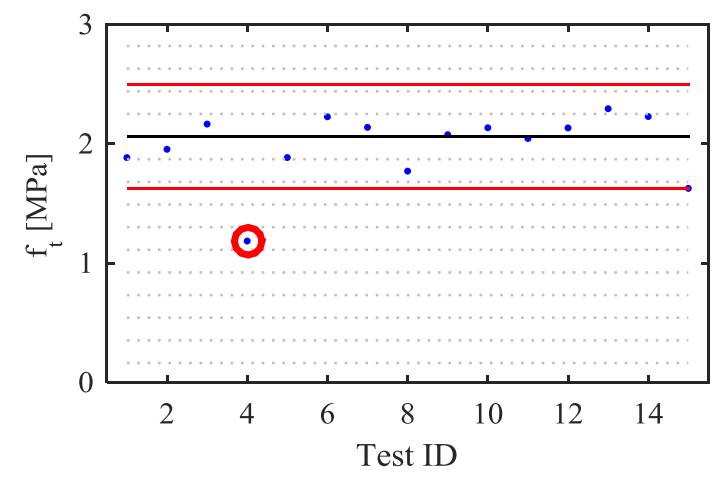

(a)

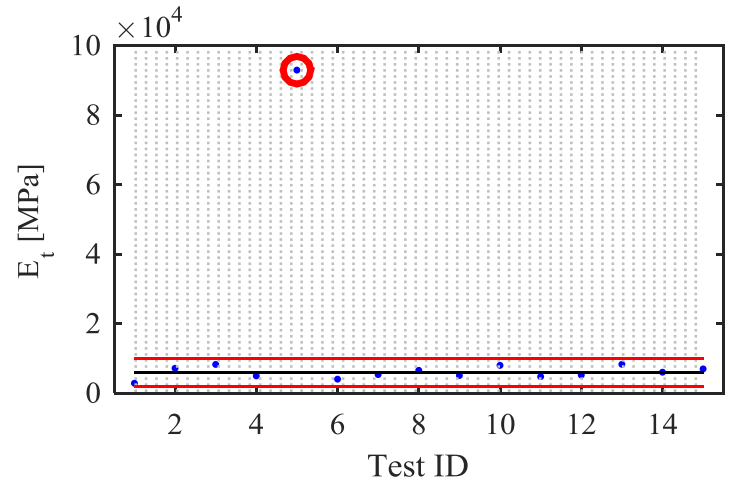

(b)

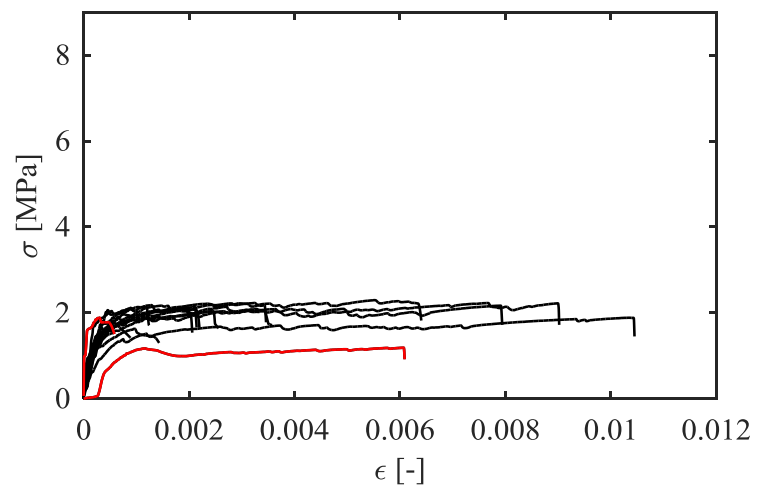

(c)

Figure 7. Outlier analysis for 15HDB L specimens in tension: (a) tensile strength outlier analysis; (b) elastic modulus outlier analysis; (c) stress strain curves (in gray) removed from the database.

After the outliers are removed from the database, the remaining curves are used in order to identify a fitting curve, which can be employed in numerical analyses involving plasterboards. A fitting curve is assessed for each test group, that is, for each board typology in each direction. Different stress-strain shapes could be used. The different methodologies adopted for tension and compression tests, respectively, are described in the following paragraphs.

\section{Tension tests}

As mentioned in the previous section, tensile tests show a stress-strain diagram which assumes a typical bilinear shape. This suggests that the stress-strain relationship can be enveloped by a bilinear curve. Four different parameters univocally define a bilinear curve (Figure 8). In this case, the initial elastic modulus $E_{t}$, the "yielding" and ultimate stresses $f_{y}$ and $f_{u}$, and the ultimate deformation $\epsilon_{u}$, are selected as the parameters. Parameters $E_{t}, f_{u}$ and $\epsilon_{u}$ are simply assessed as the average of the corresponding values resulting from the different performed tests, i.e. $E_{t, a v g}, f_{u, a v g}$ and $\epsilon_{u, a v g}$. The "yielding" stress $f_{y}$ is estimated according to an equal-energy rule: the area below the bilinear envelope is set equal to the average area $A_{\text {avg }}$ of the different tests. 


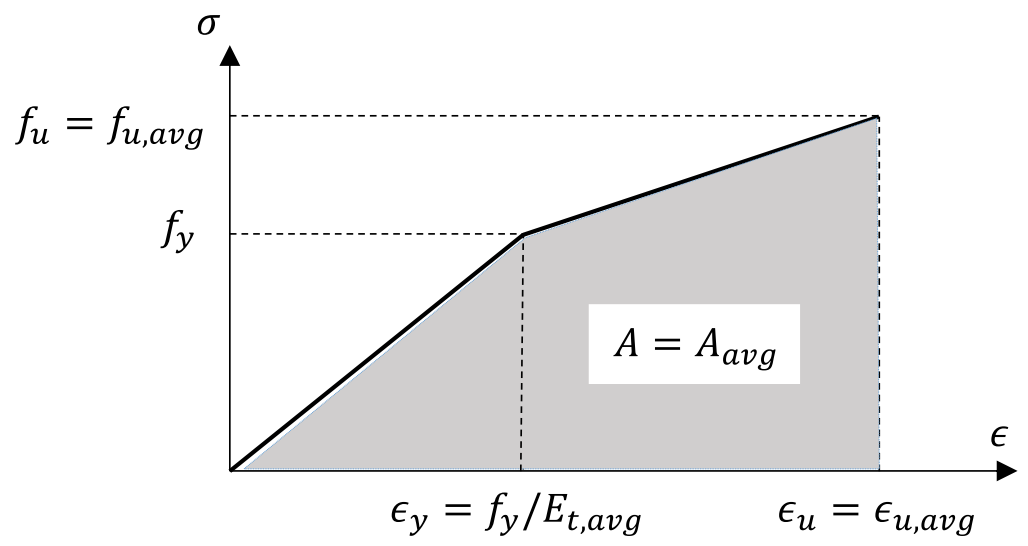

Figure 8. Bilinear envelope of the tensile stress-strain law.

From simple geometric considerations (Figure 8), it can be demonstrated that

$$
f_{y}=E_{t} \cdot\left(2 \cdot A_{\text {avg }}-f_{u} \cdot \epsilon_{u}\right) /\left(\epsilon_{u} \cdot E_{t}-f_{u}\right)
$$

which fully defines the bilinear envelope. The procedure is applied to each of the ten test groups. The resulting bilinear stress-strain curves along with the resulting parameters are included in Figure 10.

The goodness-of-fit is also assessed by means of the coefficient of determination

$$
R^{2}=1-\frac{\sum_{i}\left(\hat{y}_{i}-y_{i}\right)^{2}}{\sum_{i}\left(\bar{y}-y_{i}\right)^{2}}
$$

where $\hat{y}_{i}$ is the i-th element of the fitted model, i.e. the stresses at the different strain values, $y_{i}$ is the corresponding measured datum, $\bar{y}$ is the mean of the measured datum. The coefficient of determination ranges from 0 to 1 , where 1 indicates the best fitting.

Several comments can be drawn from the fitted stress-strain relationships of the boards in tension.

- The comparison between tension tests performed in longitudinal direction and transversal direction highlights a systematic smaller strength in transversal direction. This feature underlines the orthotropic behavior exhibited by the tested plasterboards. Moreover, a much smaller ultimate strain is exhibited in the transversal direction compared to the longitudinal direction: ultimate strain is at least twice smaller than in longitudinal direction. Finally, the elastic modulus is less influenced by the testing direction.

- The comparison between boards characterized by different thicknesses, i.e. 12SB vs $18 \mathrm{SB}$ and $12 \mathrm{HDB}$ vs $15 \mathrm{HDB}$ vs $18 \mathrm{HDB}$, underlines that the tensile strength is not clearly influenced by the thickness of the boards. Stiffness is instead generally influenced by the thickness: the larger the thickness, the larger 
the elastic modulus. Finally, the ultimate strain is also influenced by the thickness of the boards: generally the larger the thickness, the smaller the ultimate strain.

- R-squared values never exceed 0.84 ; this is probably caused by the large uncertainty associated to the tension behavior of plasterboards.

\section{Compression tests}

Compressive tests require a different approach, due to their typical stress-strain shape (Figure 6). The compressive behavior could be enveloped by a model defined by Mander et al. (1988) for the concrete compression constitutive law.

The constitutive law proposed by Mander et al. (Figure 9) is defined upon four different parameters: the maximum strength $f_{c}$, the corresponding strain $\varepsilon_{c}$, the initial elastic modulus $E_{c}$ and the ultimate strain $\varepsilon_{u}$ as follows

$$
\sigma(\epsilon)=f_{c} \cdot \frac{\epsilon}{\epsilon_{c}} \cdot \frac{r}{r-1+\left(\frac{\epsilon}{\epsilon_{c}}\right)^{r}} \text { for } 0<\epsilon<\epsilon_{u}
$$

where

$$
r=\frac{E_{c}}{E_{c}-E_{s e c}}=\frac{E_{c}}{E_{c}-\frac{f_{c}}{\epsilon_{c}}}
$$

The parameters $f_{c}, \epsilon_{c}$ and $\epsilon_{u}$ are evaluated as the mean values measured in each test group. The initial elastic modulus $E_{c}$ is estimated in order to achieve the best fitting with the experimental curves. A set of different elastic moduli is considered and the corresponding stress-strain envelopes are compared to the recorded relationships (Figure 6). A least squares approach is therefore adopted to select the elastic modulus. The procedure is applied for each board loaded in each direction in compression. The resulting fitting curves are overlapped to the experimental data in Figure 11 along with the resulting parameters of the envelope curve. Also for compression tests the goodness-of-fit is estimated by means of the coefficient of determination Rsquared. 


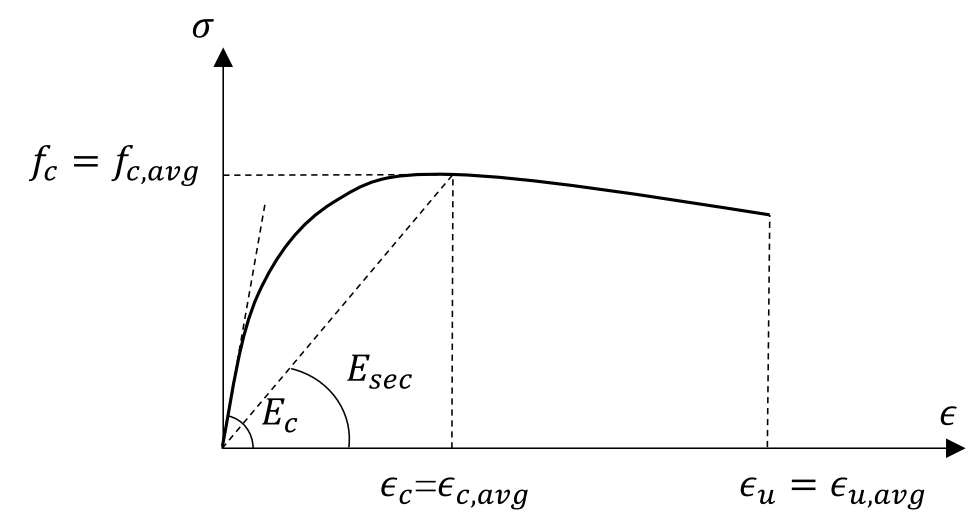

Figure 9. Adopted envelope for the compressive stress-strain law.

Several comments can be drawn from the fitted stress-strain relationships of the boards in compression.

- Compressive strength of the tested boards is in the range $3.02 \div 8.14 M P a$, whereas the elastic modulus is in the range $2130 \div 4161 \mathrm{MPa}$. The strain at which the maximum strength of the specimen is recorded, i.e. $\epsilon_{c}$, is in the vicinity of $0.25 \%$ for all the specimens, whereas the ultimate deformation is typically smaller than $0.40 \%$, except for $12 \mathrm{SB}$ boards tested in their transversal direction.

- The comparison between compression tests performed in longitudinal and transversal direction generally highlights negligible discrepancies in terms of strength, stiffness and ultimate strain. The orthotropic behavior is therefore limited to tension tests.

- The comparison between boards characterized by different thicknesses, i.e. 12SB vs $18 \mathrm{SB}$ and 12HDB vs $15 \mathrm{HDB}$ vs $18 \mathrm{HDB}$, underlines that both compression strength and stiffness are influenced by the thickness of the boards: the larger the thickness, the larger the strength and the elastic modulus.

- high-density boards exhibit larger strength and stiffness than standard boards, as shown by the comparison between 12HDB (18HDB) and 12SB (18SB) boards.

- The data are well enveloped by the selected stress-strain shape. This is clearly underlined by the Rsquared values, which are larger than 0.85 , except for $18 \mathrm{SB}$ boards tested in their longitudinal direction. 

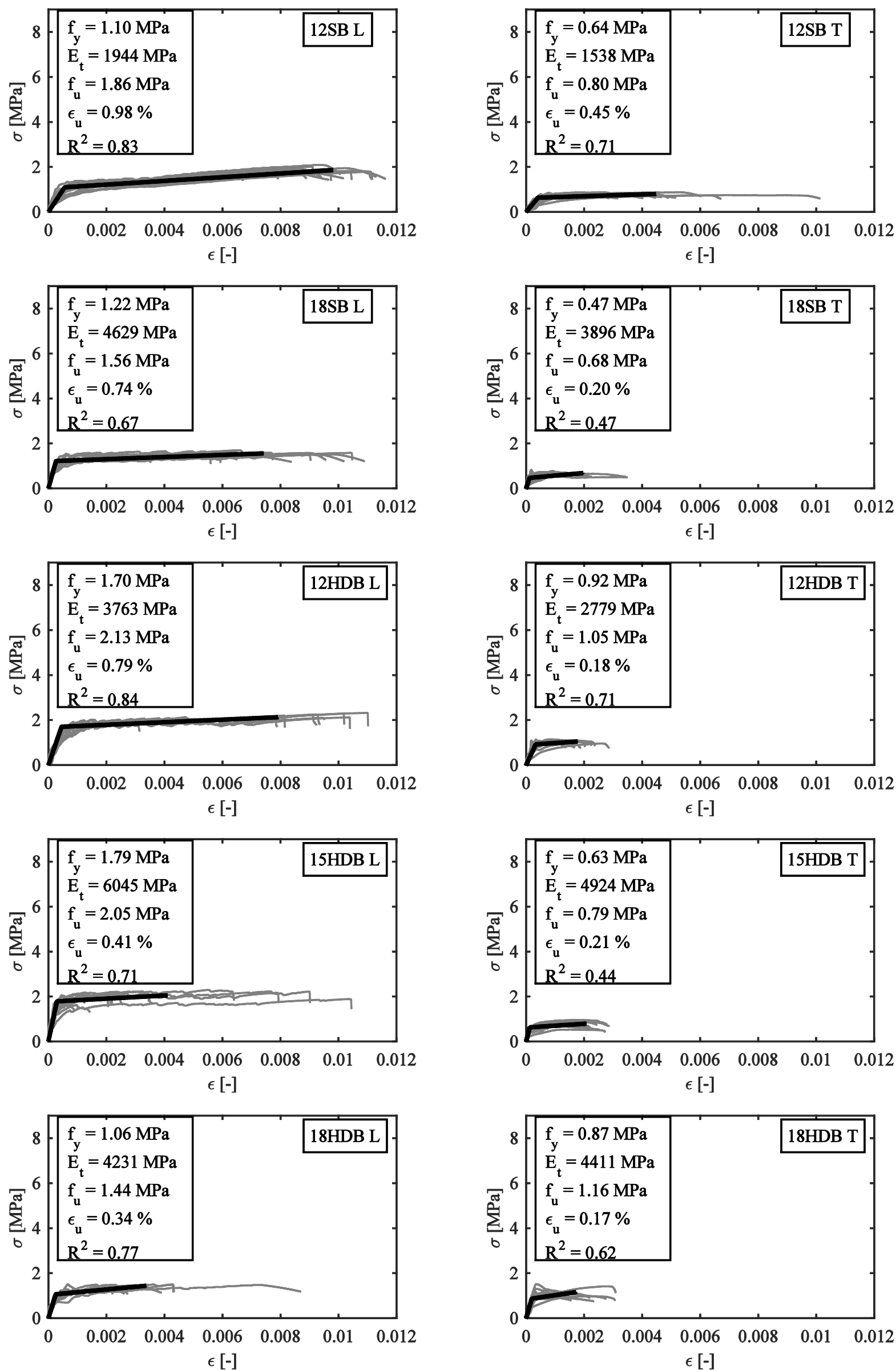

Figure 10. Tensile tests fitting for all the plasterboards in both longitudinal and transversal direction. 

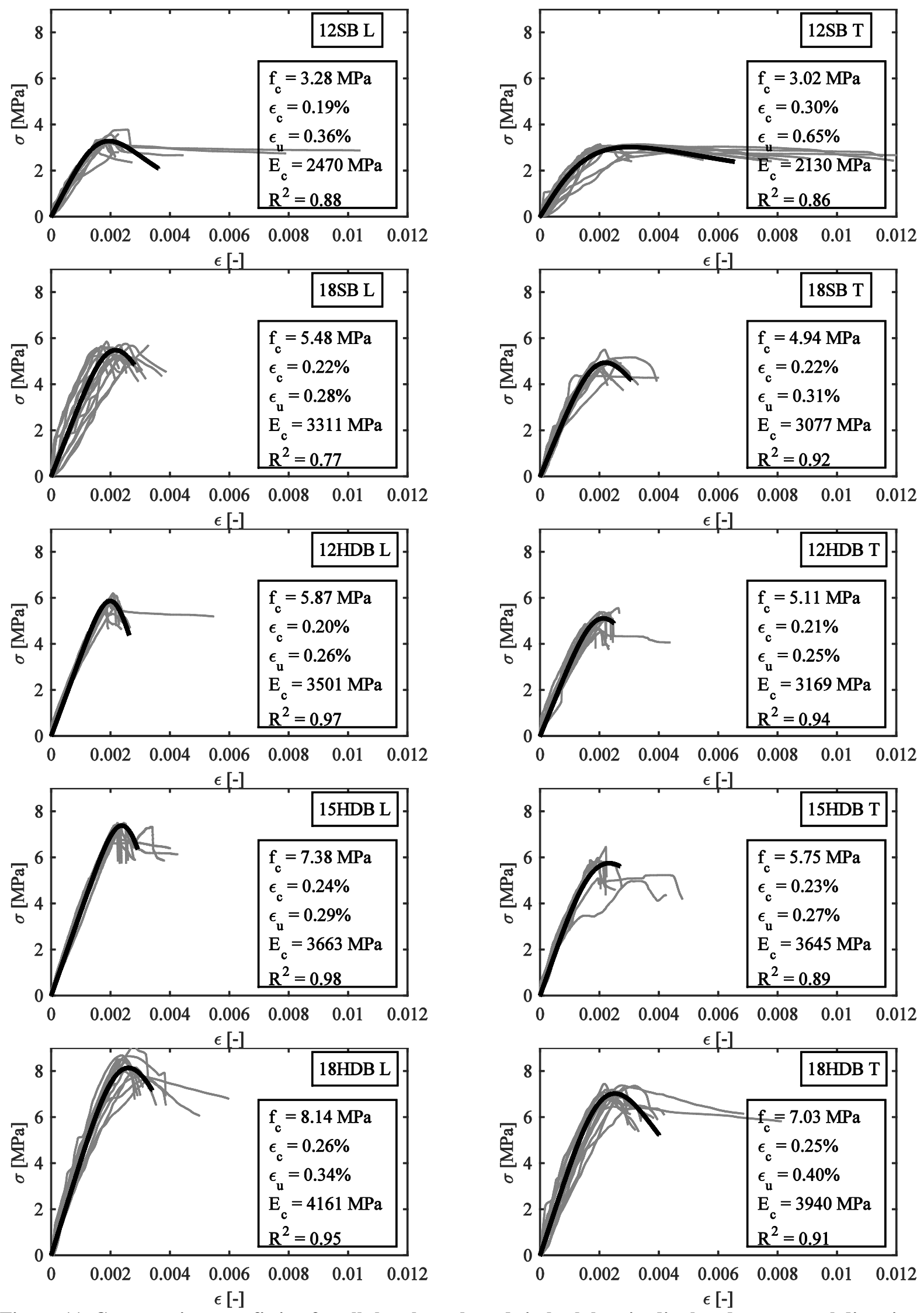

Figure 11. Compressive tests fitting for all the plasterboards in both longitudinal and transversal direction. 


\section{Statistical analysis and uncertainty assessment}

The last section of this work deals with the identification of the most suitable probability function for different mechanical characteristics of the plasterboards both in compression and in tension. Once the best probability function is assessed, the uncertainty related to these characteristics is also estimated.

A statistical analysis is performed on four parameters that are typically used in numerical analyses involving plasterboards, i.e. tensile and compressive strength, elastic moduli in compression and tension. Compressive and tensile strengths are evaluated for each test based on the maximum recorded stress; elastic moduli, instead, are evaluated according to EN 789 (CEN 2004b). The goal of this analysis is the identification of the most suitable probability function. In particular three probability distributions are considered: Normal, Lognormal and Weibull distribution. These three probability distributions are used to fit the experimental data for each parameter of the different test groups. In Figure 12 this procedure is shown only for 12SB boards loaded in their longitudinal direction, for the sake of brevity.

The Anderson-Darling test (Anderson and Darling 1952) is used to test the goodness-of-fit of Normal, Lognormal and Weibull distributions to the experimental data. This test places greater weight on the observations in the tails of the distribution, which is important for the structural safety assessment (Galasso et al. 2014). The decision to reject the null hypothesis is performed by comparing the $\mathrm{p}$-value, ranging from 0 to 1 , for the hypothesis test with the specified significance level. The test is positive, i.e. the assumed distribution cannot be rejected, if p-value is larger than the significance level, assumed equal to 5\%.

P-values for the different parameters and test groups are listed in Table 2 and Table 3. It should be also noted that p-values are typically larger than 0.05 for the different probability distribution functions. Only in few cases, the null hypothesis test is rejected, i.e. the p-values are smaller than 0.05 . 

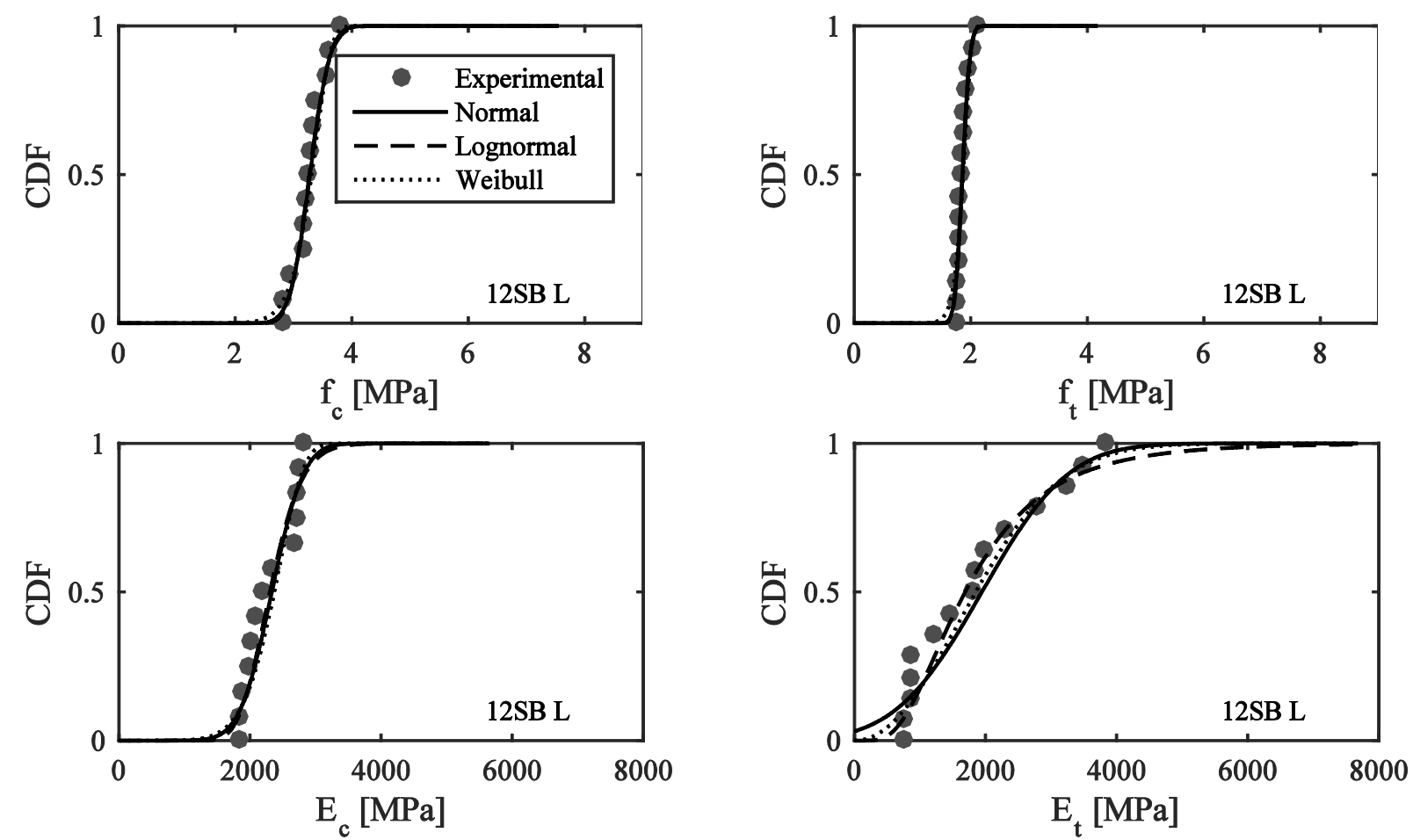

Figure 12. Fitting of experimental mechanical properties of 12SB L tests with three different probability distributions: Normal, Lognormal and Weibull distributions.

The resulting p-values allow comparing the different probability distributions for the different tests. The selected distribution functions typically exhibit similar p-values. However, Lognormal distribution is rejected only in three cases, whereas Normal and Weibull distributions are rejected in four different cases. Moreover, Lognormal distribution is typically adopted in civil structural engineering to model the uncertainty of both geometrical and mechanical properties (Celarec et al. 2012). For these reasons, Lognormal distribution is selected in the following to estimate the uncertainty associated to the selected mechanical parameters. 
Table 2. P-values obtained by Normal, Lognormal and Weibull fitting curve in tensile tests.

\begin{tabular}{|c|c|c|c|c|c|c|c|}
\hline & & \multirow{2}{*}{\multicolumn{3}{|c|}{$f_{t}$}} & \multirow{2}{*}{\multicolumn{3}{|c|}{$\mathrm{E}_{\mathrm{t}}$}} \\
\hline & & & & & & & \\
\hline & & Normal & Lognormal & Weibull & Normal & Lognormal & Weibull \\
\hline \multirow{5}{*}{ 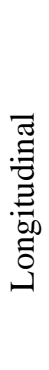 } & $12 \mathrm{SB}$ & 0.235 & 0.324 & 0.044 & 0.244 & 0.394 & 0.423 \\
\hline & $18 \mathrm{SB}$ & 0.609 & 0.510 & 0.807 & 0.087 & 0.298 & 0.065 \\
\hline & $12 \mathrm{HDB}$ & 0.938 & 0.911 & 0.843 & 0.449 & 0.296 & 0.472 \\
\hline & $15 \mathrm{HDB}$ & 0.172 & 0.089 & 0.603 & 0.817 & 0.497 & 0.764 \\
\hline & $18 \mathrm{HDB}$ & 0.077 & 0.069 & 0.091 & 0.415 & 0.590 & 0.373 \\
\hline \multirow{5}{*}{ 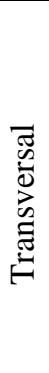 } & $12 \mathrm{SB}$ & 0.430 & 0.486 & 0.221 & 0.038 & 0.027 & 0.021 \\
\hline & 18SB & 0.835 & 0.880 & 0.603 & 0.331 & 0.229 & 0.346 \\
\hline & 12HDB & 0.194 & 0.162 & 0.287 & 0.869 & 0.151 & 0.870 \\
\hline & $15 \mathrm{HDB}$ & 0.299 & 0.098 & 0.631 & 0.146 & 0.374 & 0.304 \\
\hline & 18HDB & 0.574 & 0.778 & 0.348 & 0.053 & 0.212 & 0.068 \\
\hline
\end{tabular}

Table 3. P-values obtained by Normal, Lognormal and Weibull fitting curve in compressive tests.

\begin{tabular}{|c|c|c|c|c|c|c|c|}
\hline & & \multirow{2}{*}{\multicolumn{3}{|c|}{$f_{c}$}} & \multirow{2}{*}{\multicolumn{3}{|c|}{$\mathrm{E}_{\mathrm{c}}$}} \\
\hline & & & & & & & \\
\hline & & Normal & Lognormal & Weibull & Normal & Lognormal & Weibull \\
\hline \multirow{5}{*}{ 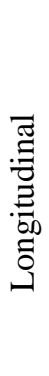 } & $12 \mathrm{SB}$ & 0.812 & 0.809 & 0.537 & 0.052 & 0.068 & 0.030 \\
\hline & 18SB & 0.040 & 0.035 & 0.070 & 0.024 & 0.163 & 0.060 \\
\hline & 12HDB & 0.906 & 0.891 & 0.803 & 0.256 & 0.294 & 0.139 \\
\hline & 15HDB & 0.739 & 0.730 & 0.689 & 0.674 & 0.459 & 0.912 \\
\hline & 18HDB & 0.532 & 0.504 & 0.554 & 0.231 & 0.562 & 0.151 \\
\hline \multirow{5}{*}{ 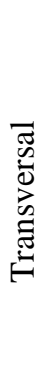 } & $12 \mathrm{SB}$ & 0.396 & 0.350 & 0.745 & 0.959 & 0.438 & 0.968 \\
\hline & $18 \mathrm{SB}$ & 0.829 & 0.819 & 0.570 & 0.767 & 0.904 & 0.585 \\
\hline & 12HDB & 0.024 & 0.020 & 0.035 & 0.761 & 0.915 & 0.498 \\
\hline & 15HDB & 0.146 & 0.113 & 0.191 & 0.174 & 0.363 & 0.083 \\
\hline & 18HDB & 0.510 & 0.431 & 0.843 & 0.552 & 0.853 & 0.370 \\
\hline
\end{tabular}

Uncertainty in the mechanical properties of structural materials may jeopardize the seismic performance of buildings, e.g. concrete variability for RC structures (De Stefano et al. 2014, 2015). The uncertainty associated 
to each of the four selected parameters of plasterboards is therefore evaluated considering the corresponding lognormal distribution functions. In particular, both median $x_{m}$ and dispersion $\beta$ values of the distribution functions are used for the comparison (Table 4).

The comments included in previous sections can be also drawn from the comparison of median values for the different tests. Moreover, Table 4 highlights rather similar median elastic modulus values both in compression and in tension. Median tensile strength, instead, is typically smaller than compressive strength.

The dispersion of the data around the median value is significantly influenced by the considered mechanical parameter. In particular, elastic modulus in tension is characterized by a large uncertainty, i.e. $\beta$ values up to 0.68. Both tensile and compressive strengths show small variability around the mean. Finally, the uncertainty is influenced neither by the direction of loading nor by the thickness of the boards.

Table 4. Parameters of lognormal distributions which fit the experimental data for the different test groups.

\begin{tabular}{|c|c|c|c|c|c|c|c|c|c|}
\hline & & \multicolumn{2}{|c|}{$f_{t}$} & \multicolumn{2}{|c|}{$\mathrm{E}_{\mathrm{t}}$} & \multicolumn{2}{|c|}{$\mathrm{f}_{\mathrm{c}}$} & \multicolumn{2}{|c|}{$E_{c}$} \\
\hline & & $x_{m}[M P a]$ & $\beta[-]$ & $x_{m}[M P a]$ & $\beta[-]$ & $x_{m}[M P a]$ & $\beta[-]$ & $x_{m}[M P a]$ & $\beta[-]$ \\
\hline \multirow{5}{*}{ 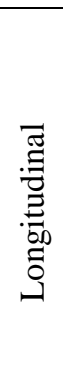 } & $12 \mathrm{SB}$ & 1.86 & 0.05 & 1687 & 0.56 & 3.27 & 0.08 & 2303 & 0.17 \\
\hline & $18 \mathrm{SB}$ & 1.55 & 0.06 & 4508 & 0.24 & 5.47 & 0.05 & 3608 & 0.46 \\
\hline & $12 \mathrm{HDB}$ & 2.12 & 0.06 & 3736 & 0.13 & 5.86 & 0.04 & 3464 & 0.05 \\
\hline & $15 \mathrm{HDB}$ & 2.04 & 0.10 & 5801 & 0.31 & 7.38 & 0.01 & 3779 & 0.09 \\
\hline & $18 \mathrm{HDB}$ & 1.44 & 0.05 & 4143 & 0.21 & 8.13 & 0.06 & 4539 & 0.21 \\
\hline \multirow{5}{*}{ 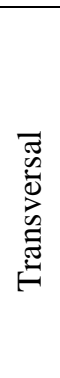 } & $12 \mathrm{SB}$ & 0.80 & 0.06 & 1362 & 0.54 & 3.02 & 0.03 & 1779 & 0.31 \\
\hline & $18 \mathrm{SB}$ & 0.68 & 0.11 & 3300 & 0.66 & 4.93 & 0.06 & 3459 & 0.15 \\
\hline & $12 \mathrm{HDB}$ & 1.04 & 0.07 & 2634 & 0.37 & 5.10 & 0.07 & 3150 & 0.14 \\
\hline & $15 \mathrm{HDB}$ & 0.78 & 0.19 & 4052 & 0.68 & 5.74 & 0.07 & 3609 & 0.15 \\
\hline & 18HDB & 1.15 & 0.15 & 4228 & 0.30 & 7.02 & 0.05 & 3723 & 0.21 \\
\hline
\end{tabular}

\section{CONCLUSIONS}

Plasterboard components are widely used in current buildings worldwide. Plasterboards are typically employed for partitions, wall lining and ceilings. Despite their extensive use, the lack of a comprehensive test campaign on plasterboards in the current literature is denoted. An extensive test campaign, consisting of 302 tests, is therefore performed aiming at evaluating compression and tension behavior of plasterboards. A set of five 
plasterboard typologies is selected, considering different board thicknesses and both standard and high-density boards. Both tensile and compression tests are performed according to EN 789. The tests are performed in two different load directions, i.e. parallel or transversal to the direction of production.

The comparison of the stress-strain relationships in compression and in tension shows that:

- a ductile behavior is exhibited in tension along with a more brittle behavior in compression; the ultimate tensile strain is much larger than the ultimate compressive strain. Moreover, tensile strength is systematically smaller than compressive strength. Tension behavior is significantly different from compression behavior, mainly due to the presence of the paper; indeed, the contribution of the paper is significant just in case the specimen is loaded in tension;

- smaller tensile strength is recorded in the transversal direction compared to the longitudinal direction, clearly underlining the orthotropic behavior exhibited by plasterboards; compression behavior is not much influenced by testing direction, since the paper contribution is negligible in case the specimen is loaded in compression;

- the comparison among boards with different thicknesses highlights that the larger the thickness, the larger the compressive strength;

Some outliers in the recorded data are clearly visible in many stress-strain plots. They may have been caused by some errors in the preparation of the specimen, in the application of loads, etc.. At this purpose, an outlier analysis is preliminary applied to the experimental data.

Regression laws that can be employed to model both compression and tension behavior of plasterboards are defined for future implementations of the actual stress-strain relationships in different applications, e.g. FEM analysis of shear stud wall panels. A bilinear stress-strain envelope is adopted for tensile behavior, whereas a model typically used for concrete is selected for compression behavior.

Some additional comments can be drawn from the fitted stress-strain relationships.

- The strain at which the maximum compression strength of the specimen is recorded occurs at about $0.25 \%$, whereas the ultimate deformation is typically smaller than $0.40 \%$. 
- Smaller tensile ultimate strain is exhibited in the transversal direction compared to the longitudinal direction. The tensile elastic modulus is less influenced by the testing direction, exhibiting similar values in the two orthogonal directions.

- Tensile strength is not clearly influenced by the board thickness, whereas compression strength and both tensile and compressive stiffness are influenced by board thickness.

- High-density boards generally exhibit both larger stiffness and strength than standard boards both in tension and in compression.

- Experimental data are well enveloped by the selected stress-strain shapes in compression, whereas a worse fit is obtained in tension.

Finally the most suitable probability function, modeling the maximum strength and the elastic modulus both in compression and in tension, is assessed. Lognormal distribution is selected based on Anderson-Darling test. The uncertainty associated to each of the four selected parameters is therefore evaluated considering the corresponding lognormal distribution functions. The dispersion of the data around the median value is significantly influenced by the considered mechanical parameter. In particular, elastic modulus in tension is characterized by a large uncertainty, i.e. $\beta$ values up to 0.68 . Both tensile and compressive strengths show small variability around the mean. Finally, the uncertainty is influenced neither by the direction of loading nor by the thickness of the boards.

The estimated parameters can be used as reference material for future numerical and probabilistic research studies involving plasterboard components which can be represented by the tested specimens; it is therefore suggested to carefully check the properties of the considered plasterboards, e.g. the density of gypsum plaster, before considering any of the results reported herein. Moreover, caution should be taken in generalizing the outcomes of this research study, since all tested components were produced by a single manufacturer.

\section{ACKNOWLEDGEMENTS}

This study was partially funded by the Italian Department of Civil Protection in the frame of the national project DPC-ReLUIS 2015 RS8. The contribution of Eng. Luigi Giannetti in the analysis of the experimental data is acknowledged. The authors also thank Mrs Raffaelina Divano, an English language expert, for the paper proofreading. 


\section{REFERENCES}

Anderson, T. W., Darling, D. A. (1952). "Asymptotic Theory of Certain "Goodness of Fit" Criteria Based on Stochastic Processes." 193-212

Arjunan, A., Wang, C. J., Yahiaoui, K., Mynors, D. J., Morgan, T., English, M. (2013). "Finite element acoustic analysis of a steel stud based double-leaf wall." Build Environ 67, 202-210

Asiz, A., Chui, Y. H., Doudak, G., Ni, C., Mohammad, M. (2011). "Contribution of Plasterboard Finishes to Structural Performance of Multi-storey Light Wood Frame Buildings." Procedia Engineer 14, $1572-$ 1581

Bouterf, A., Roux, S., Hild, F., Vivier, G., Brajer, X., Maire, E., Meille, S. (2015). "Damage law identification from full field displacement measurement: Application to four-point bending test for plasterboard." European Journal of Mechanics - A/Solids 49, 60-66

Celarec, D., Ricci, P., Dolšek, M. (2012). "The sensitivity of seismic response parameters to the uncertain modelling variables of masonry-infilled reinforced concrete frames." Eng Struct 35(0), 165-177

CEN (2004a) EN 520: Gypsum plasterboards - Definitions, requirements and test methods. Brussels, Belgium CEN (2004b) EN 789: Determination of mechanical properties of wood-based panels. Brussels, Belgium

Cimbala, J. M. (2011) Outliers (available at https://www.mne.psu.edu/me345/Lectures/outliers.pdf). Penn State University

De Stefano, M., Tanganelli, M., Viti, S. (2014). "Variability in concrete mechanical properties as a source of in-plan irregularity for existing RC framed structures." Eng Struct 59, 161-172

De Stefano, M., Tanganelli, M., Viti, S. (2015). "Seismic performance sensitivity to concrete strength variability: A case-study." Earthq Struct 9(2), 321-337

Ellis, B. R., Bougard, A. J. (2001). "Dynamic testing and stiffness evaluation of a six-storey timber framed building during construction." Eng Struct 23(10), 1232-1242

Eurogypsum (2014) Living with Gypsum: From Raw Material to Finished Products. Available at http://www.eurogypsum.org/_Uploads/dbsAttachedFiles/livingwithgypsum.pdf.

Galasso, C., Maddaloni, G., Cosenza, E. (2014). "Uncertainly Analysis of Flexural Overstrength for Capacity Design of RC Beams." J Struct Eng 140(7), 04014037 
Gencel, O., del Coz Diaz, J. J., Sutcu, M., Koksal, F., Alvarez Rabanal, F. P., Martinez-Barrera, G., Brostow, W. (2014). "Properties of gypsum composites containing vermiculite and polypropylene fibers: Numerical and experimental results." Energy and Buildings 70, 135-144

Lee, T. H., Kato, M., Matsumiya, T., Suita, K., Nakashima, M. (2007). "Seismic performance evaluation of non-structural components: Drywall partitions." Earthq Eng Struct Dyn 36(3), 367-382

Magliulo, G., Petrone, C., Capozzi, V., Maddaloni, G., Lopez, P., Manfredi, G. (2014). "Seismic performance evaluation of plasterboard partitions via shake table tests." Bull Earthq Eng 12(4), 1657-1677

Magliulo, G., Petrone, C., Capozzi, V., Maddaloni, G., Lopez, P., Talamonti, R., Manfredi, G. (2012). "Shake Table Tests on Infill Plasterboard Partitions." Open Constr Build Technol J 6(Suppl 1-M10), 155-163

Mander, J., Priestley, M., Park, R. (1988). "Theoretical Stress-Strain Model for Confined Concrete." J Struct Eng 114(8), 1804-1826

Memari, A., Solnosky, R. (2014). "In-Plane Shear Performance of Wood-Framed Drywall Sheathing Wall Systems under Cyclic Racking Loading." Open Journal of Civil Engineering 4(1), 54-70

Oliver-Ramirez, A., Garcia-Santos, A., Neila-Gonzalez, F. J. (2011). "Physical and mechanical characterization of gypsum boards containing phase change materials for latent heat storage." Mater Construcc 61(303), 465-484

Peterman, K., Schafer, B. (2014). "Sheathed Cold-Formed Steel Studs under Axial and Lateral Load." J Struct Eng 140(10), 04014074

Petrone, C., Magliulo, G., Lopez, P., Manfredi, G. (2015a). "Out-of-plane seismic performance of plasterboard partitions via quasi-static tests." Bulletin of the New Zealand Society for Earthquake Engineering (under review)

Petrone, C., Magliulo, G., Lopez, P., Manfredi, G. (2015b). "Seismic fragility evaluation of plasterboard partitions via in-plane quasi-static tests." Earthq Eng Struct Dyn (online early)

Retamales, R., Davies, R., Mosqueda, G., Filiatrault, A. (2013). "Experimental Seismic Fragility of ColdFormed Steel Framed Gypsum Partition Walls." J Struct Eng 139(8), 1285-1293

Serrette, R., Encalada, J., Juadines, M., Nguyen, H. (1997). "Static Racking Behavior of Plywood, OSB, Gypsum, and FiberBond Walls with Metal Framing." J Struct Eng 123(8), 1079-1086 
Serrette, R., Ogunfunmi, K. (1996). "Shear Resistance of Gypsum-Sheathed Light-Gauge Steel Stud Walls." J Struct Eng 122(4), 383-389

Telue, Y., Mahendran, M. (2004). "Behaviour and design of cold-formed steel wall frames lined with plasterboard on both sides." Eng Struct 26(5), 567-579 